\title{
Enhancing the critical current in quasiperiodic pinning arrays below and above the matching magnetic flux
}

\author{
V. R. Misko, ${ }^{1,2}$ D. Bothner, ${ }^{3}$ M. Kemmler,${ }^{3}$ R. Kleiner, ${ }^{3}$ D. Koelle, ${ }^{3}$ F. M. Peeters, ${ }^{1}$ and Franco Nori ${ }^{2,4}$ \\ ${ }^{1}$ Departement Fysica, Universiteit Antwerpen, B-2020 Antwerpen, Belgium \\ ${ }^{2}$ Advanced Science Institute, RIKEN, Wako-shi, Saitama 351-0198, Japan \\ ${ }^{3}$ Physikalisches Institut-Experimentalphysik II and Center for Collective Quantum Phenomena, Universität Tübingen, \\ D-72076 Tübingen, Germany \\ ${ }^{4}$ Physics Department, University of Michigan, Ann Arbor, Michigan 48109-1040, USA
}

(Received 2 July 2010; published 8 November 2010)

\begin{abstract}
Quasiperiodic pinning arrays, as recently demonstrated theoretically and experimentally using a fivefold Penrose tiling, can lead to a significant enhancement of the critical current $I_{c}$ as compared to "traditional" regular pinning arrays. However, while regular arrays showed only a sharp peak in $I_{c}(\Phi)$ at the matching flux $\Phi_{1}$ and quasiperiodic arrays provided a much broader maximum at $\Phi<\Phi_{1}$, both types of pinning arrays turned out to be inefficient for fluxes larger than $\Phi_{1}$. We demonstrate theoretically and experimentally the enhancement of $I_{c}(\Phi)$ for $\Phi>\Phi_{1}$ by using non-Penrose quasiperiodic pinning arrays. This result is based on a qualitatively different mechanism of flux pinning by quasiperiodic pinning arrays and could be potentially useful for applications in superconducting microelectronic devices operating in a broad range of magnetic fields.
\end{abstract}

DOI: $10.1103 /$ PhysRevB.82.184512

PACS number(s): 74.25.Sv, 74.25.Wx, 74.78.-w

\section{INTRODUCTION}

Pinning of Abrikosov vortices in superconductors is a topic that has attracted considerable interest, both with respect to the fundamental physical properties of so-called "vortex-matter" and with respect to device applications. In the latter case, for bulk or thick film superconductors, the inclusion of nanodefects, acting as pinning sites, typically randomly distributed within the superconductors, has been shown to significantly enhance the critical current density, which is important for increasing the current-carrying capacity of wires and tapes, e.g., for applications in superconducting magnets (see, e.g., Ref. 1 and references therein). On the other hand, recent progress in the fabrication of nanostructures has provided a wide variety of well-controlled lithographically defined artificial pinning sites, and experiments can control both the location and the strength of each pinning center in vortex-confining arrays of pinning sites (APS) (see, e.g., Refs. 2-5). In this context it has been shown that, e.g., microholes (antidots) or magnetic dots may improve the performance of microelectronic thin-film devices. Examples are the suppression of low-frequency flux noise in superconducting quantum interference devices by strategically positioned antidots ${ }^{6,7}$ or the enhancement of the quality factor of superconducting microwave resonators operated in magnetic fields, which can be achieved by incorporating antidot arrays into the thin-film structures. ${ }^{8}$ From a basic point of view, it is interesting to explore the optimum pinning landscape provided by APS. One convenient way to do this is to investigate the critical current $I_{c}$ versus magnetic flux $\Phi$ for different arrangements of pinning sites.

Periodic artificial APS were proposed as devices able of trapping magnetic flux and thus enhancing $I_{c}$. However, the efficiency of periodic APS is strongly limited to so-called matching fields, i.e., when the number of vortices is commensurate with the number of pins, thus resulting in strong but very narrow peaks in the critical current versus magnetic flux, $I_{c}(\Phi)$. This shortcoming can be overcome by using more sophisticated pinning topologies, e.g., quasiperiodic (QP) APS. ${ }^{9-14}$ In particular, using a fivefold Penrose tiling (see, e.g., Refs. 15-21) as an APS (i.e., placing pins on the vertices of a Penrose tiling) results in a considerable enhancement of $I_{c}(\Phi)$, i.e., significant broadening of the peak in $I_{c}(\Phi)$, as was recently demonstrated theoretically ${ }^{9,10}$ and experimentally. ${ }^{12,13}$ The underlying idea of using QP APS is that, contrary to regular APS, they include many periodicities and thus can trap vortices for magnetic fluxes other than matching values $\Phi_{i}$. As shown for Penrose-tiling APS, $, 10,12,13$ in addition to the matching-flux peak at $\Phi$ $=\Phi_{1}, I_{c}(\Phi)$ acquires a remarkably broad maximum for $\Phi$ $<\Phi_{1}$. This maximum turned out to be even more robust with respect to variations in parameters of APS than the sharp peak at $\Phi=\Phi_{1} \cdot{ }^{9,10}$ However, in theory and experiments, $I_{c}(\Phi)$ decreases rapidly for fluxes $\Phi>\Phi_{1}$ for QP Penrosetiling APS as well as for regular APS. Here, we discuss the essential difference in the flux pinning for magnetic fluxes below and above the matching value $\Phi=\Phi_{1}$ and we demonstrate, theoretically and experimentally, how the critical current $I_{c}(\Phi)$ can be improved in the important regime where $\Phi>\Phi_{1}$, by using novel artificial APS.

The paper is organized as follows: the model and experiment are described in Secs. II and III, respectively. In Sec. $\mathrm{IV}$, we analyze the enhancement of the critical current below the first matching field in different aperiodic pinning arrays and compare the results to those for a fivefold Penrose-tiling APS. The mechanisms of vortex "conductivity" leading to the enhancement of the critical current below and above the matching flux are discussed in Sec. V. In Sec. V, we also present our experimental and numerical results for QP APS demonstrating the enhancement of the critical current for $\Phi$ $>\Phi_{1}$. Finally, the conclusions are given in Sec. VI. 


\section{SIMULATION}

We model a three-dimensional slab, infinitely long in the $z$ direction, by a two-dimensional (2D) (in the $x y$ plane) simulation cell with periodic boundary conditions. Note that following Refs. 9 and 10 we apply periodic boundary conditions at the boundaries of the square simulation cell while the quasiperiodic array is taken smaller than the cell. This is done in order to prevent imposing ad hoc periodicity to the vortex motion in a QP APS: the free-of-pinning region between the QP APS and the boundary of the simulation cell serves as a reservoir of vortices that mimics the external applied magnetic field. This reservoir (of thickness on the order of few intervortex distances) erases the memory, i.e., the information on the coordinate of a vortex leaving the cell. Each additional vortex enters the QP sample at the current point of "least resistance to entry," similarly to the way vortices enter any other sample when placed in an external magnetic field. This approach has been successfully used in numerous simulations in the past (see, e.g., Refs. 9, 10, and 22-24).

To study the dynamics of vortex motion, we perform simulated annealing simulations by numerically integrating the overdamped equations of motion (see, e.g., Refs. 9 and 10 for a description of the method),

$$
\eta \mathbf{v}_{i}=\mathbf{f}_{i}=\mathbf{f}_{i}^{v v}+\mathbf{f}_{i}^{v p}+\mathbf{f}_{i}^{T}+\mathbf{f}_{i}^{d} .
$$

Here $\mathbf{f}_{i}$ is the total force per unit length acting on vortex $i, \mathbf{f}_{i}^{v v}$ and $\mathbf{f}_{i}^{v p}$ are the forces due to vortex-vortex and vortex-pin interactions, respectively, $\mathbf{f}_{i}^{T}$ is the thermal stochastic force, and $\mathbf{f}_{i}^{d}$ is the driving force; $\mathbf{v}_{i}$ is the velocity and $\eta$ is the viscosity. All the forces are expressed in units of $f_{0}$ $=\Phi_{0}^{2} / 8 \pi^{2} \lambda^{3}$, where $\Phi_{0}=h c / 2 e$, and lengths (fields) are in units of $\lambda\left(\Phi_{0} / \lambda^{2}\right)$.

Following Refs. 9 and 10, we model vortex pinning by short-range parabolic potential wells located at positions $\mathbf{r}_{k}^{(p)}$. The pinning force is

$$
\mathbf{f}_{i}^{v p}=\sum_{k}^{N_{p}}\left(\frac{f_{p}}{r_{p}}\right)\left|\mathbf{r}_{i}-\mathbf{r}_{k}^{(p)}\right| \Theta\left(\frac{r_{p}-\left|\mathbf{r}_{i}-\mathbf{r}_{k}^{(p)}\right|}{\lambda}\right) \hat{\mathbf{r}}_{i k}^{(p)},
$$

where $N_{p}$ is the number of pinning sites, $f_{p}$ is the maximum pinning force of each potential well, $r_{p}$ is the range of the pinning potential, $\Theta$ is the Heaviside step function, and $\hat{\mathbf{r}}_{i k}^{(p)}$ $=\left(\mathbf{r}_{i}-\mathbf{r}_{k}^{(p)}\right) /\left|\mathbf{r}_{i}-\mathbf{r}_{k}^{(p)}\right|$.

The ground state of a system of moving vortices is obtained by simulating the field-cooled experiments (e.g., Ref. $25)$. For deep short-range ( $\delta$-like) potential wells, the energy required to depin vortices trapped by pinning sites is proportional to the number of pinned vortices, $N_{v}^{(p)}$. Therefore, in this approximation, we can define the normalized critical current as follows: ${ }^{9,10}$

$$
I_{c}(\Phi)=j_{0} \frac{N_{v}^{(p)}(\Phi)}{N_{v}(\Phi)},
$$

and study the dimensionless value $J_{c}=I_{c} / j_{0}$, where $N_{v}$ is the total number of vortices within the simulation cell of the area $A$, and $j_{0}$ is a constant (i.e., the current needed to depin $N_{v}$ noninteracting vortices, $\left.j_{0}=N_{v} j_{v}^{d p}\right)$. Since the area of the QP pattern $A_{q p}$ is less than $A$, the critical current Eq. (3) acquires a prefactor $A_{q p} / A \approx 0.58$ (Refs. 9, 10, and 26) in the simulations below. We use narrow potential wells as pinning sites, with the maximum pinning force $f_{p}$ and the radius $r_{p}$ $=0.04 \lambda$ to $0.1 \lambda$.

\section{EXPERIMENT}

Experiments were performed on $d=60$-nm-thick $\mathrm{Nb}$ films containing circular antidots (diameter $D=400 \mathrm{~nm}$ ) at the vertices of various types of QP APS (see insets of Figs. 1 and 2) in an area of $200 \times 200 \mu \mathrm{m}^{2}$. The average antidot density is $n_{p} \approx 0.5 \mu \mathrm{m}^{-2}$, corresponding to a first matching field $B_{1}=n_{p} \Phi_{0} \approx 1 \mathrm{mT}$. Electric transport measurements for the determination of the critical current $I_{c}(\Phi)$ of perforated $\mathrm{Nb}$ bridges were performed at well-controlled temperatures $T$ and magnetic fields; for details of sample fabrication and experimental setup see Refs. 12 and 27. The bridges had transition temperatures $T_{c} \approx 8.4 \mathrm{~K}$ and transition widths varying from 15 to $25 \mathrm{mK}$. The temperature dependence of the critical current at $B=0$ differed from bridge to bridge leading to different absolute values of $I_{c}(B=0)$ at the same reduced temperatures $T / T_{c}$. The experimental data shown below, were recorded at $T / T_{c}=0.9995$, where the absolute values of $I_{c}$ for different samples were very similar. Note that according to our estimates based on a simple core pinning model (see Ref. 12), the maximum value of the critical depinning current in quasiperiodic pinning arrays of antidots reached the values of about 0.5 of the Ginzburg-Landau depairing current, for the used parameters.

\section{CRITICAL CURRENT IN QP TILING APS}

For a fivefold Penrose-tiling APS, ${ }^{9,10,12,13}$ pinning arrays with many periodicities ${ }^{28}$ can enhance the critical current ${ }^{29}$ for a broader range of magnetic fluxes as compared to periodic APS. In order to examine and possibly further optimize $J_{c}(\Phi)$ in APS, in this section we study the critical current as a function of applied magnetic flux, for different QP tilings.

First, we consider a tiling consisting of the simplest shapes, squares and triangles (both compatible with the lowest-energy vortex lattices). The result for a quasiperiodic square-triangle tiling ${ }^{30}$ is shown in Fig. 1(a). For comparison, Fig. 1 also shows the $J_{c}(\Phi)$ dependence for a fivefold Penrose-tiling APS. $J_{c}(\Phi)$ for the QP square-triangle tiling has a broad maximum at $\Phi_{m}<\Phi_{1}$, which is formed due to the rearrangement of vortices between the square and triangular tiles when their density changes. Note the absence of a peak at $\Phi_{1}$, indicating that the local matching conditions between the APS and the vortex lattice are not fulfilled at the given parameters of the APS (i.e., the average distance between the pinning sites, their maximum strength and radius). However, a peak in $J_{c}(\Phi)$ at $\Phi_{1}$ is observed for the Penrosetiling APS. Another tiling consisting of the same simple shapes (i.e., squares and triangles) is the nonpisot squaretriangular tiling ${ }^{30}$ shown in the inset of Fig. 1(b). Although these two types of tilings are characterized by different inflation rules (i.e., different arrangements of the tiles), they contain the same elements, i.e., hexagons formed by six tri- 

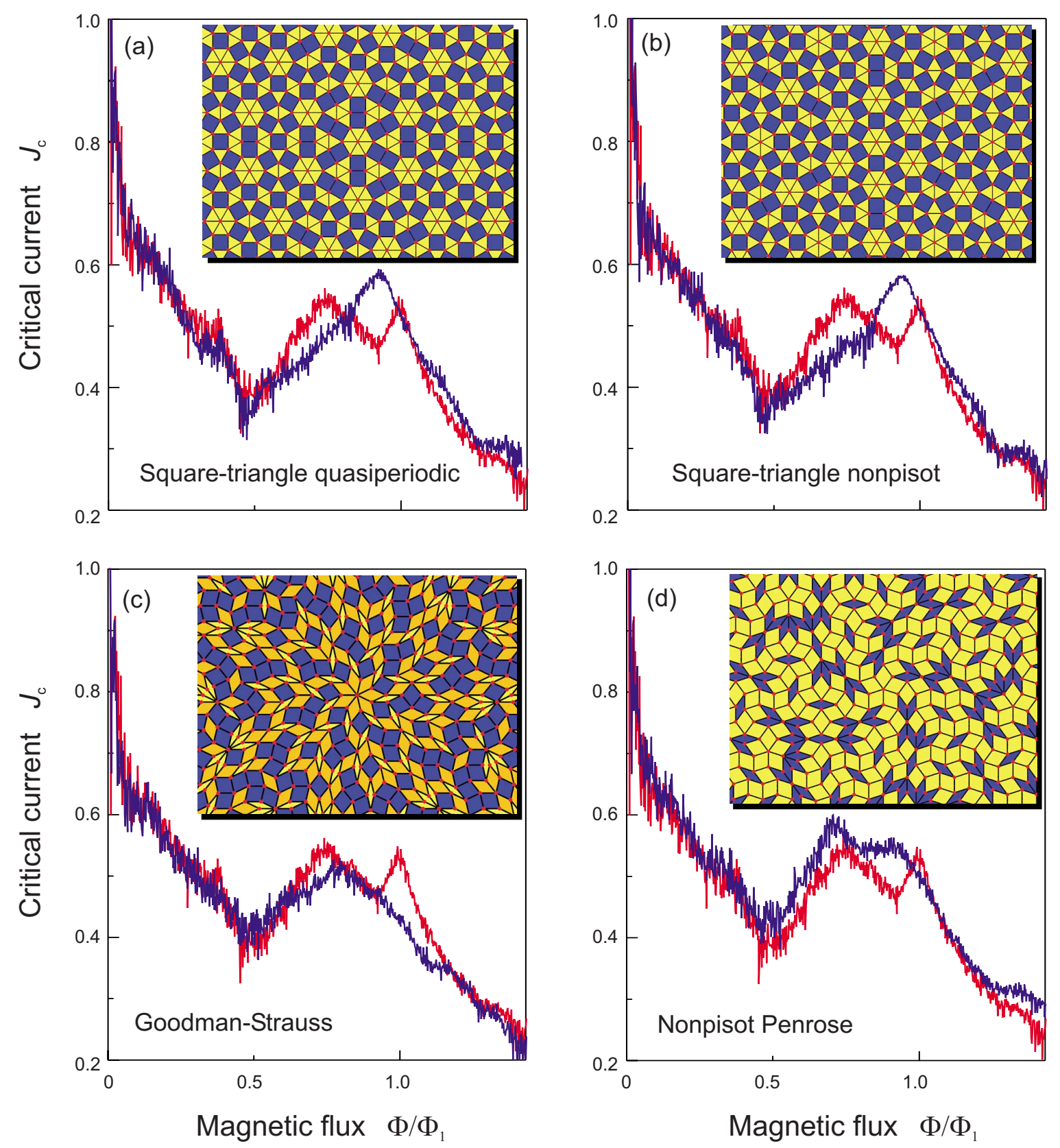

FIG. 1. (Color online) Normalized critical current, $J_{c}$ versus normalized magnetic flux, $\Phi / \Phi_{1}\left(\propto\right.$ number of vortices $N_{v}$ ), for different QP APS [shown by solid blue (dark gray) lines]: (a) square-triangle quasiperiodic tiling, (b) square-triangle nonpisot tiling, (c) Goodman-Strauss tiling, and (d) nonpisot Penrose tiling. Insets show the corresponding tiling patterns. For comparison, the function $J_{c}\left(\Phi / \Phi_{1}\right)$ for a fivefold Penrose-tiling APS is shown by a red (gray) line. The $J_{c}\left(\Phi / \Phi_{1}\right)$ curves are shown for $f_{p} / f_{0}=2$ and $r_{p} / \lambda=0.1$. These values of $f_{p}$ and $r_{p}$ are also used in Figs. 2-4.

angles surrounded by six squares. This similarity in the structure determines similar local matching effects between vortices and the pinning centers for these two tilings. As a result, the $J_{c}(\Phi)$ curve for the nonpisot square-triangular tiling [Fig. 1(b)] turns out to be practically identical to that for the quasiperiodic square-triangular tiling [compare to Fig. 1(a)]. An example of a tiling consisting of three different tiles (three types of rhombuses) is a Goodman-Strauss tiling ${ }^{21}$ shown in the inset of Fig. 1(c). Surprisingly, the behavior of $J_{c}(\Phi)$ for this APS is very similar to that of the Penrose-tiling APS, although it has an obvious shortcoming: it does not contain the maximum at the matching flux $\Phi_{1}$ [Fig. 1(c)].

These examples indicate that the considered tilings provide, in general, a weaker enhancement of the critical current as compared to the fivefold Penrose-tiling APS which seems to be the best among these competitors. Let us then consider a different modification of a Penrose tiling, namely, a nonpisot Penrose tiling. ${ }^{21}$ This tiling also consists of two types of rhombuses. It is shown in the inset of Fig. 1(d). As shown in the main panel of Fig. 1(d), this APS provides an even better enhancement of $J_{c}(\Phi)$ within nearly the same, as for the fivefold Penrose tiling, range of magnetic flux: it is charac- 


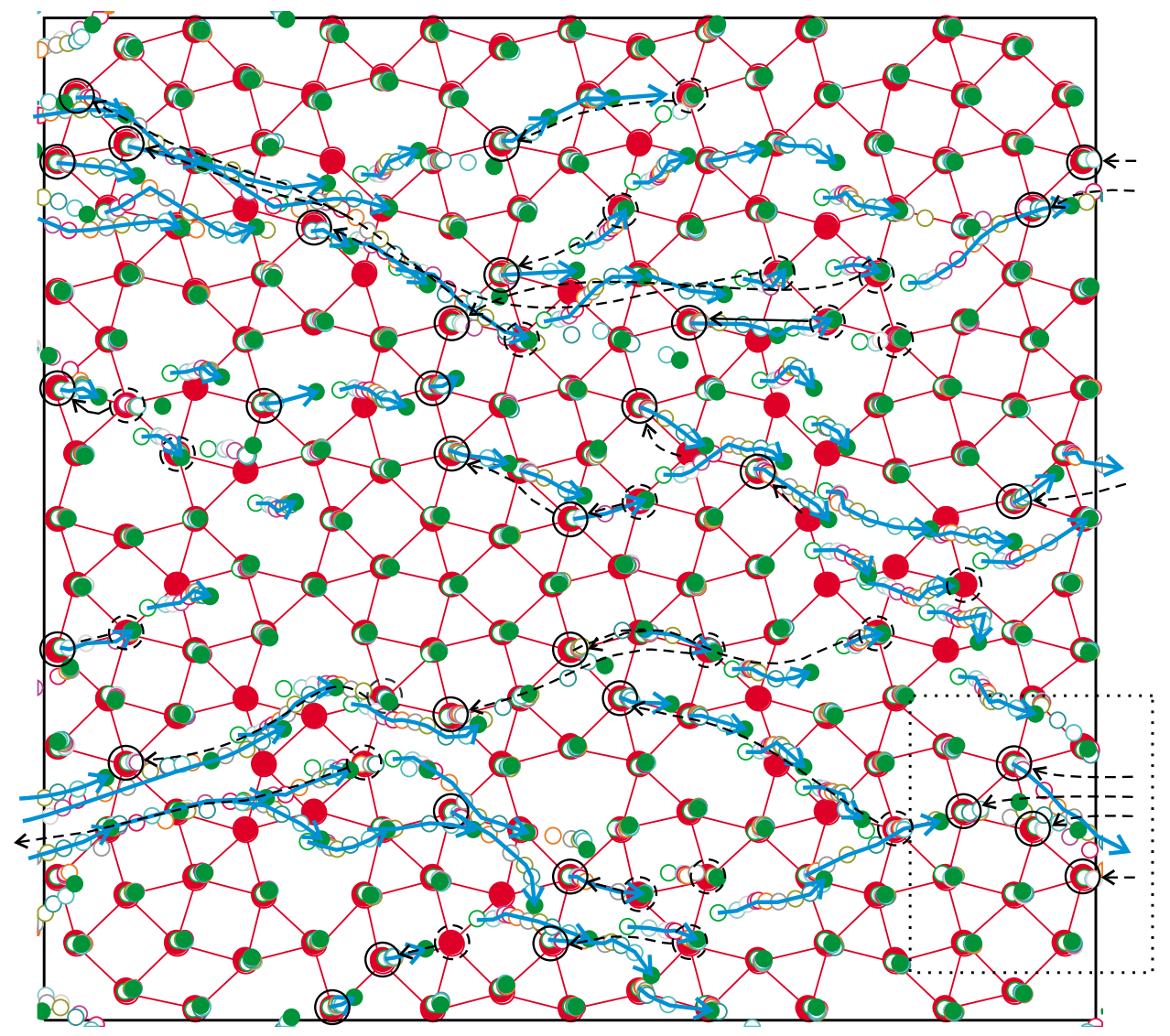

FIG. 2. (Color online) Flow pattern for vortices driven by an external current illustrating the holelike vortex conductivity in the Shield-tiling APS for $\Phi \leqslant \Phi_{1}: \Phi \approx 0.95 \Phi_{1}$. The pinning sites are shown by filled large red (gray) circles. Vortex positions are shown for ten successive time intervals $t_{i}$, where $i=1-10$ : by open small circles, for $i=1-9$, and by filled small green (gray) circles, for $i=10$. The Lorentz force drives vortices from the left to the right. Most of the vortices do not move: their positions just slightly change (polarized) with respect to the centers of the pinning sites. The trajectories of moving vortices (from the left to the right) are shown by thick blue (gray) arrows. The motion of vortices results in effective propagation of vacancies (holes) from the right to the left (shown by thin dashed black arrows), including holes entering the sample from outside, as in the region marked by the dotted rectangle. Newly created holes are shown by thin solid black circles while annihilated holes (i.e., filled by vortices) are shown by thin dashed black circles.

terized by an almost-flat plateau within the broad maximum in $J_{c}(\Phi)$. This improvement of the pinning properties is explained by the specific arrangement of the rhombuses in this kind of tiling, which turns out to be closer to the energetically favorable arrangement of vortices in the lattice, thus providing better vortex-pin matching conditions for various densities. For the fivefold Penrose-tiling APS, ${ }^{9,10,12,13}$ the maximum of the critical current at $\Phi=\Phi_{1}$ is separated from the main broad maximum and is not as stable as the broad maximum with respect to changes in the parameters of the APS (i.e., the maximum pinning strength). ${ }^{9,10}$ The advantage of the APS based on the nonpisot Penrose tiling is that it provides a smooth transition from the matching-field configuration (i.e., at $\Phi=\Phi_{1}$ ) to lower-density pinned states (i.e., when some of the vertices of the small tiles become unoccupied $\left.^{9,10}\right)$. This provides a flattening of the $J_{c}(\Phi)$ curve [Fig. 1(d)] and thus increases the overall value of the maximum $J_{c}$ within the range of the plateaulike maximum. This important improvement could be useful for applications of QP APS.

\section{V. "HOLELIKE" AND "ELECTRONLIKE" VORTEX CONDUCTIVITY}

As can be seen in Fig. 1, all the above results obtained using different APS, while providing different profiles of $J_{c}(\Phi)$, still have a common feature: $J_{c}$ drops drastically for $\Phi>\Phi_{1}$ as it does in periodic APS (for both cases, $\Phi>\Phi_{1}$ and $\Phi<\Phi_{1}$ ).

Can we improve this? One might think that in practice we can increase the density of the pinning sites in the array and thus trap flux for any $\Phi$. Indeed this might work to some extent but the efficiency of an APS, especially QP APS, is determined not only by the ratio of the average intervortex distance to the average period of the APS $\left(d_{\mathrm{av}}^{\mathrm{APS}}\right)$ but also by the ratio of those distances to the magnetic field penetration depth $\lambda$, which is specific for a given superconductor. To understand this, let us now briefly recall the essential difference between vortex lattice pinning by periodic and quasiperiodic APS. In the former case, if the symmetry of the vortex lattice coincides with that of the pinning array (e.g., for a triangular pinning array; in case of a square APS, transitions 

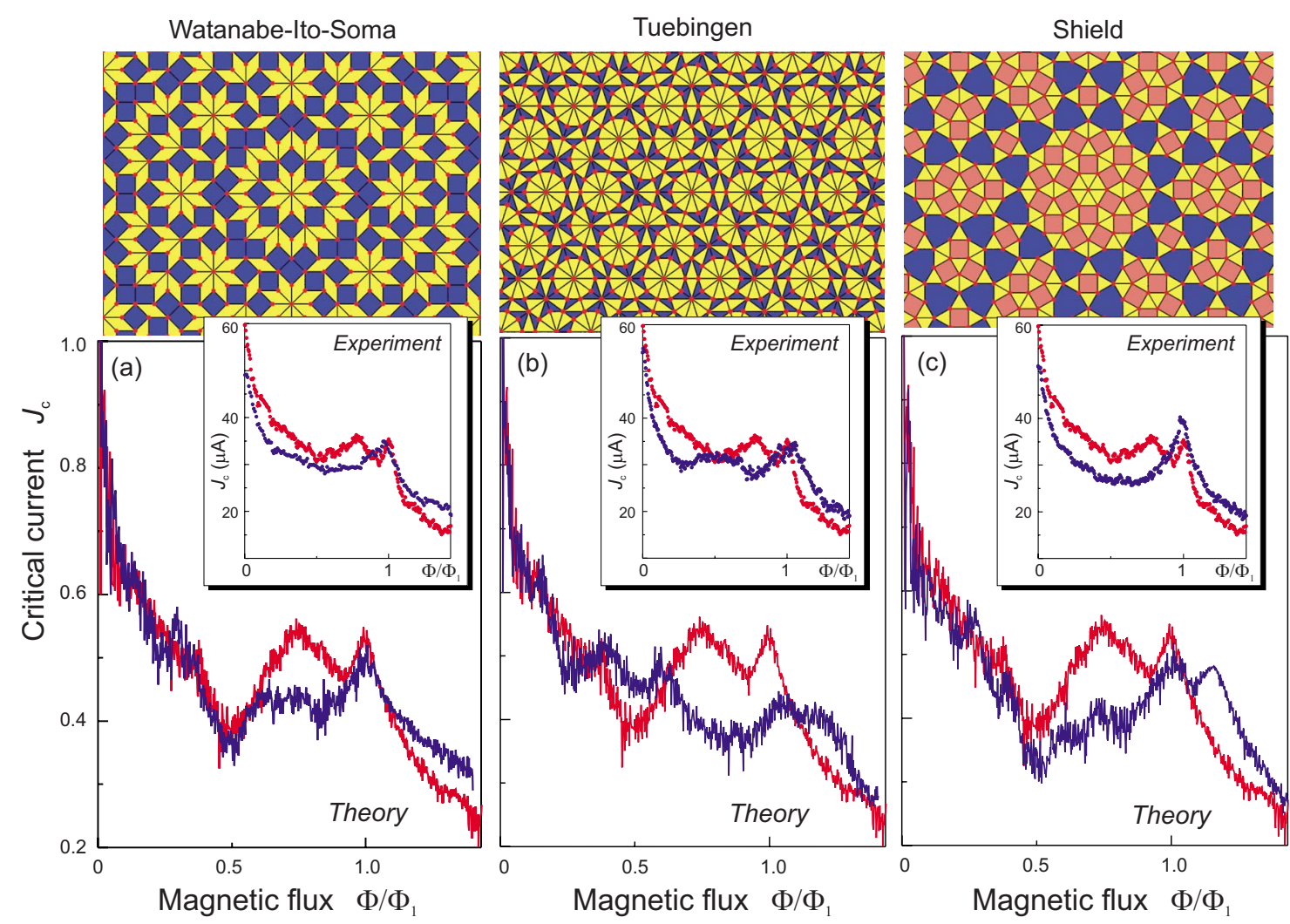

FIG. 3. (Color online) Normalized critical current (experiment and theory), $J_{c}$ versus normalized magnetic flux, $\Phi / \Phi_{1}(\propto$ number of vortices $N_{v}$ ), for different QP APS [solid blue (dark gray) line]: (a) Watanabe-Ito-Soma tiling, (b) Tuebingen tiling, and (c) Shield tiling. Insets show the corresponding tiling patterns. The function $J_{c}\left(\Phi / \Phi_{1}\right)$ for a fivefold Penrose-tiling APS is shown by red (gray) line, for comparison.

between the triangular, half-pinned, and square lattices occur-see, e.g., Ref. 31) the vortex lattice and the pinning array are commensurate for any $d_{\mathrm{av}}^{\mathrm{APS}} / \lambda$. In contrast to periodic APS, the pinning efficiency of a QP APS is sensitive to this ratio $d_{\mathrm{av}}^{\mathrm{APS}} / \lambda$ since the elastic deformations of the vortex lattice are involved in its collective pinning by QP APS.

This is explained by the fact that a vortex lattice is incommensurate with any QP tiling (see Refs. 9 and 10). Thus, the efficiency of a QP APS does not scale with the density of pinning sites. On the other hand, shifting the broad maximum in $J_{c}(\Phi)$ toward larger values of $\Phi$, i.e., $\Phi>\Phi_{1}$, results in the loss of pinning efficiency for $\Phi<\Phi_{1}$. Moreover, the problem of the effective pinning for magnetic flux for $\Phi$ $>\Phi_{1}$ is of fundamental interest and, as we show below, is related to a qualitatively different type of flux pinning in aperiodic APS.

The fast drop of $J_{c}(\Phi)$ for $\Phi>\Phi_{1}$ is explained by the enhanced mobility of interstitial vortices, which is rather high even in QP APS. Note that a very low concentration of interstitial vortices (as compared to pinned vortices) is sufficient to drastically reduce the critical current. Using the language of conductivity in metals and semiconductors, we can call the motion of interstitial vortices "electronlike vortex conductivity." Correspondingly, the situation $\Phi<\Phi_{1}$ when there are vacancies, i.e., unoccupied pinning sites, is similar to hole-type conductivity, since in this case the motion of vacancies is similar to the motion of holes in semiconductors. For fluxes $\Phi \leq \Phi_{1}$, the concentration of "holes" is low but the "vortex conductivity" is still provided by the motion of vortices (Fig. 2). However, in contrast to periodic arrays where this motion is possible due to the channeling of vortices [leading to the drop of $J_{c}(\Phi)$ for $\Phi<\Phi_{1}$ ], the process of channeling is strongly suppressed in QP systems (as in metallic quasicrystals where the usual periodic Bloch solution for electrons does not exist ${ }^{32}$ ). Thus this can explain the presence of the maxima and the fast drop of $J_{c}(\Phi)$ in QP APS, for $\Phi<\Phi_{1}$ and for $\Phi>\Phi_{1}$, correspondingly.

Therefore, the problem is how to immobilize the additional (i.e., electronlike) vortices for $\Phi>\Phi_{1}$. Although in a QP APS they appear to be less mobile than in periodic APS (no Bloch-type solutions), they can jump to adjacent tiles (hopping conductivity) or "push" pinned vortices and thus unpin them producing secondary interstitial vortices. To prevent this scenario, we propose to immobilize extra vortices in a special way, namely, to create well-separated "reservoirs" for them which themselves are arranged in a quasiperiodic order. This will prevent the extra vortices from (i) hopping-type motion (due to the isolation of the reservoirs) and (ii) channeling (due to the QP order).

Among 2D tilings, the Watanabe-Ito-Soma tiling ${ }^{21,33}$ (consisting of squares and rhombuses) and Tuebingen tiling ${ }^{34}$ (consisting of two types of triangles) shown in the insets of Figs. 3(a) and 3(b), respectively, are possible candidates. These tilings contain isolated "islands" formed by triangles and rhombuses and the density of pinning sites on these islands is rather high. The plots of $J_{c}(\Phi)$ in Figs. 3(a) and 3(b) 

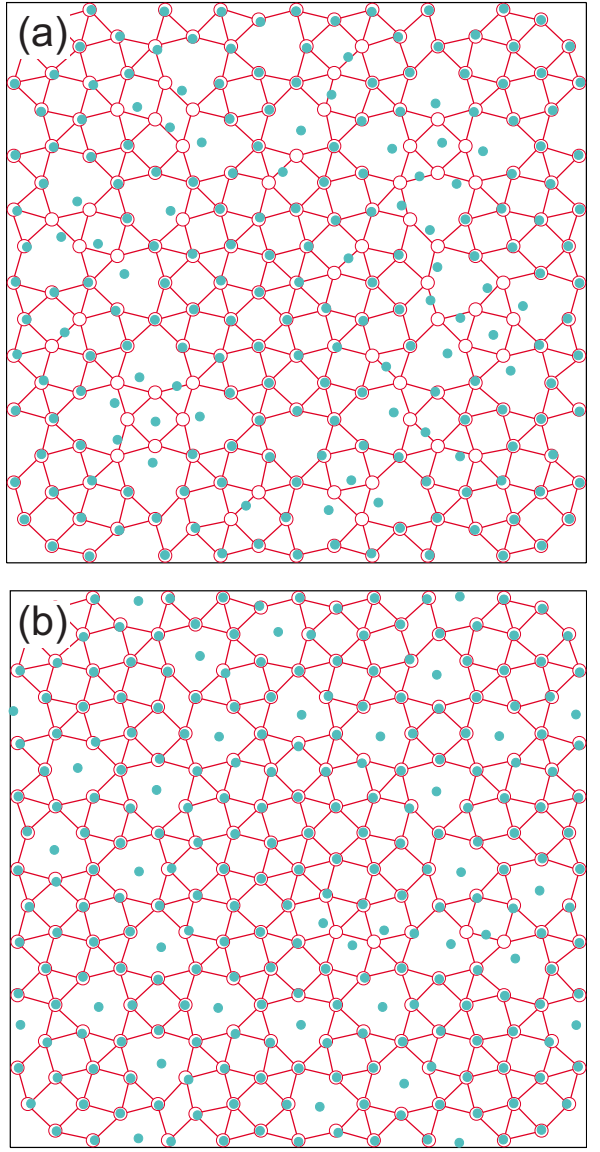

FIG. 4. (Color online) Distribution of vortices in a Shield-tiling APS calculated for: $\Phi=\Phi_{1}$ (a) and $\Phi=1.18 \Phi_{1}$ (b). The vortices are shown by green (light gray) solid dots while the pinning sites by red (gray) open circles.

show that indeed there is an appreciable increase in the critical current for $\Phi>\Phi_{1}$, although the overall value of $J_{c}$ for $\Phi>\Phi_{1}$ for both tilings is rather low. These features of $J_{c}(\Phi)$ can be seen in both experimental and theoretical $J_{c}(\Phi)$ curves. Note that the experimental curves in Figs. 3(a)-3(c) qualitatively [and to a certain extent quantitatively-see Figs. 3(a) and 3(b)] reproduce the features under discussion of the calculated $J_{c}(\Phi)$ curves, i.e., the enhancement of $J_{c}(\Phi)$ for $\Phi>\Phi_{1}$. A more detailed quantitative comparison will require additional analysis of $J_{c}(\Phi)$ for different parameters of pinning arrays (e.g., radii of the antidots) and various temperatures. Such analysis is beyond the scope of the present work and will be reported elsewhere. ${ }^{35}$

A significant enhancement of $J_{c}(\Phi)$ for $\Phi>\Phi_{1}$ is obtained with the Shield tiling ${ }^{21,36}$ (with squares, triangles, and threefold hexagons) [Fig. 3(c)]. The large tiles are excellent traps for interstitial vortices (see Fig. 4). These tiles are well separated (i.e., do not have common sides) and are arranged in a QP order [see inset of Fig. 3(c)]. Thus, $J_{c}(\Phi)$ obtains a pronounced maximum of the same magnitude as the maximum at the first matching flux $\Phi_{1}$. (However the broad maximum for $\Phi<\Phi_{1}$ is suppressed, compared to the fivefold Penrose-tiling APS.)

\section{CONCLUSIONS}

The critical depinning current $J_{c}(\Phi)$ was analyzed theoretically and experimentally for different quasiperiodic-tiling arrays of pinning sites. We showed that a fivefold Penrosetiling APS provides stronger enhancement of $J_{c}(\Phi)$ than other 2D QP tilings for a broad range of the applied magnetic flux. It was demonstrated that the pinning properties of the Penrose-tiling APS can be improved by using a different modification of the tiling, i.e., nonpisot Penrose tiling. This leads to an overall higher value of $J_{c}$ for a broader range of magnetic fields. We proposed a mechanism for the flux pinning in QP APS which implies an effective trap and space separation of interstitial vortices. This prevents hopping-type motion and channeling of vortices in the APS and thus results in an increase in the critical current for flux values larger than the matching flux $\Phi_{1}$. These results might be useful for future applications in microelectronic devices requiring a high $J_{c}$ over a broad range of the applied flux. Our proposal can be easily extended, mutatis mutandi, to other related systems, including colloidal suspensions interacting with pinning traps provided by arrays of optical tweezers ${ }^{37}$ or vortices in rotating Bose-Einstein condensates pinned by potential landscapes created by corotating lasers. ${ }^{38}$

\section{ACKNOWLEDGMENTS}

This work was supported by the "Odysseus" Program of the Flemish Government and the Flemish Science Foundation (FWO-V1), the Interuniversity Attraction Poles (IAP) Programme-Belgian State-Belgian Science Policy, the FWO-Vl, and by the DFG via SFB/TRR21. V.R.M. is grateful to the FWO-V1 for the support of the research stay at the DML (ASI, RIKEN), and to F.N. for hospitality. M.K. gratefully acknowledges support from the Carl-Zeiss-Stiftung, and D.B. from the Evangelisches Studienwerk e.V. Villigst. F.N. acknowledges partial support from the Laboratory of Physical Sciences, National Security Agency, Army Research Office, DARPA, AFOSR, National Science Foundation under Grant No. 0726909, JSPS-RFBR under Contract No. 09-0292114, Grant-in-Aid for Scientific Research (S), MEXT Kakenhi on Quantum Cybernetics, and Funding Program for Innovative R\&D on S\&T (FIRST).
${ }^{1}$ J. Gutiérrez, A. Llordés, J. Gázquez, M. Gibert, N. Romà, S. Ricart, A. Pomar, F. Sandiumenge, N. Mestres, T. Puig, and X. Obradors, Nature Mater. 6, 367 (2007).

${ }^{2}$ V. V. Moshchalkov, M. Baert, V. V. Metlushko, E. Rosseel, M. J.
Van Bael, K. Temst, R. Jonckheere, and Y. Bruynseraede, Phys. Rev. B 54, 7385 (1996).

${ }^{3}$ J. E. Villegas, S. Savel'ev, F. Nori, E. M. Gonzalez, J. V. Anguita, R. García, and J. L. Vicent, Science 302, 1188 (2003). 
${ }^{4}$ R. Wördenweber, P. Dymashevski, and V. R. Misko, Phys. Rev. B 69, 184504 (2004).

${ }^{5}$ V. R. Misko, S. Savel'ev, A. L. Rakhmanov, and F. Nori, Phys. Rev. Lett. 96, 127004 (2006); Phys. Rev. B 75, 024509 (2007).

${ }^{6}$ P. Selders and R. Wördenweber, Appl. Phys. Lett. 76, 3277 (2000).

${ }^{7}$ P. Lahl and R. Wördenweber, Appl. Phys. Lett. 81, 505 (2002).

${ }^{8}$ P. Bushev, D. Bothner, J. Nagel, M. Kemmler, K. Konovalenko, A. Loerincz, K. Ilin, M. Siegel, D. Koelle, R. Kleiner, and F. Schmidt-Kaler, arXiv:1009.3425 (unpublished).

${ }^{9}$ V. Misko, S. Savel'ev, and F. Nori, Phys. Rev. Lett. 95, 177007 (2005).

${ }^{10}$ V. R. Misko, S. Savel'ev, and F. Nori, Phys. Rev. B 74, 024522 (2006).

${ }^{11}$ J. E. Villegas, M. I. Montero, C.-P. Li, and I. K. Schuller, Phys. Rev. Lett. 97, 027002 (2006)

${ }^{12}$ M. Kemmler, C. Gürlich, A. Sterck, H. Pöhler, M. Neuhaus, M. Siegel, R. Kleiner, and D. Koelle, Phys. Rev. Lett. 97, 147003 (2006).

${ }^{13}$ A. V. Silhanek, W. Gillijns, V. V. Moshchalkov, B. Y. Zhu, J. Moonens, and L. H. A. Leunissen, Appl. Phys. Lett. 89, 152507 (2006).

${ }^{14}$ R. B. G. Kramer, A. V. Silhanek, J. Van de Vondel, B. Raes, and V. V. Moshchalkov, Phys. Rev. Lett. 103, 067007 (2009).

${ }^{15}$ R. Penrose, Math. Intell. 2, 32 (1979).

${ }^{16}$ D. Levine and P. J. Steinhardt, Phys. Rev. Lett. 53, 2477 (1984).

${ }^{17}$ H. C. Jeong and P. J. Steinhardt, Phys. Rev. B 55, 3520 (1997).

${ }^{18}$ P. J. Steinhardt, H.-C. Jeong, K. Saitoh, M. Tanaka, E. Abe, and A. P. Tsai, Nature (London) 396, 55 (1998).

${ }^{19}$ Quasicrystals: The State of the Art, 2nd ed., edited by P. J. Steinhardt and D. P. Divincenzo (World Scientific, Singapore, 1999).

${ }^{20}$ Quasicrystals, edited by J.-B. Suck, M. Schreiber, and P. Häussler (Springer, Berlin, 2002).

${ }^{21}$ B. Grünbaum and G. C. Shephard, Tilings and Patterns (W.H. Freeman, New York, 1987).

${ }^{22}$ R. A. Richardson, O. Pla, and F. Nori, Phys. Rev. Lett. 72, 1268 (1994).

${ }^{23}$ F. Nori, Science 271, 1373 (1996).

${ }^{24}$ C. J. Olson, C. Reichhardt, and F. Nori, Phys. Rev. B 56, 6175 (1997).
${ }^{25}$ K. Harada, O. Kamimura, H. Kasai, T. Matsuda, A. Tonomura, and V. V. Moshchalkov, Science 274, 1167 (1996).

${ }^{26}$ Small variation in this factor is related to slightly different numbers of pinning sites which fit within area $A_{q p}$ for different QP tilings.

${ }^{27}$ M. Kemmler, D. Bothner, K. Ilin, M. Siegel, R. Kleiner, and D. Koelle, Phys. Rev. B 79, 184509 (2009).

${ }^{28}$ Note that while QP APS contain an infinite number of periodicities, the corresponding diffraction patterns in the reciprocal $k$ space form a QP hierarchical self-similar set of $\delta$ peaks of decreasing intensity. As a result, only few periodicities turn out to be significant in QP APS.

${ }^{29} \mathrm{We}$ would like to emphasize that using various quasiperiodic pinning arrays we increase the critical current $J_{c}(\Phi)$ in regions below $\left(\Phi<\Phi_{1}\right)$ and above the matching flux $\left(\Phi>\Phi_{1}\right)$ where $J_{c}(\Phi)$ decreases very rapidly in periodic APS. Thus by broadening the maximum in $J_{c}(\Phi)$ we significantly enhance the critical current as compared to the case of periodic APS. However, we do not increase the magnitude of the $J_{c}(\Phi)$ peak at $\Phi=\Phi_{1}$.

${ }^{30}$ R. Paredes, J. L. Aragón, and R. A. Barrio, Phys. Rev. B 58, 11990 (1998).

${ }^{31}$ W. V. Pogosov, V. R. Misko, H. J. Zhao, and F. M. Peeters, Phys. Rev. B 79, 014504 (2009).

${ }^{32}$ B. Freedman, G. Bartal, M. Segev, R. Lifshitz, D. N. Christodoulides, and J. W. Fleischer, Nature (London) 440, 1166 (2006); Shahar Even-Dar Mandel and R. Lifshitz, arXiv:0808.3659 (unpublished).

${ }^{33}$ Y. Watanabe, M. Ito, and T. Soma, Acta Crystallogr., Sect. A: Found. Crystallogr. 43, 133 (1987).

${ }^{34}$ M. Baake, P. Kramer, M. Schlottmann, and D. Zeidler, Int. J. Mod. Phys. B 4, 2217 (1990).

${ }^{35}$ D. Bothner, M. Kemmler, V. R. Misko, F. M. Peeters, F. Nori, R. Kleiner, and D. Koelle, (unpublished).

${ }^{36}$ F. Gähler, in Quasicrystalline Materials, edited by Ch. Janot and J. M. Dubois (World Scientific, Singapore, 1988).

${ }^{37}$ J. C. Crocker and D. G. Grier, J. Colloid Interface Sci. 179, 298 (1996).

${ }^{38}$ S. Tung, V. Schweikhard, and E. A. Cornell, Phys. Rev. Lett. 97, 240402 (2006). 